\title{
CREATION OF IMAGE MODELS FOR INSPECTING VISUAL FLAWS ON CAPACITIVE TOUCH SCREENS
}

\author{
Yuan-Shyi Peter Chiu, Hong-Dar Lin* \\ Department of Industrial Engineering and Management, Chaoyang University of Technology,Wufong District, Taiwan
}

Touch screens (TSs) are commonly applied in many electronic appliances such as smartphones, tablets, etc. Currently, capacitive touch screens (CTSs) are the main touch technology of screen panels due to many excellent electronic properties. Problems exist in inspecting flaws inlaid in appearances of CTSs with structural patterns. Area flaws are a type of common visual defect that comprises dust, bubbles, ripple marks, and other flaws of bigger sizes. These flaws have the attributes of low contrast, brightness with slow changes, unusual and non-orientation forms, and sometimes both bright and dark flaws existing at the same time in a region. This paper suggests image models based on transformation filtering to inspect the area flaws on appearances of CTSs. We apply the Haar wavelet transform with flat zone filtering technique to eliminate the structural patterns of background by means of filtering an approximate sub-image of a breakdown wavelet domain image. Subsequently, the filtered image is reversely transformed to obtain a rebuilt image in spatial domain. Last, the rebuilt image with intensified flaws can be simply partitioned into three species (black flaws, gray flaws, and white background) by using a statistical interval estimation method. Therefore, the intricate area flaws are precisely identified by the suggested scheme. We contrast our approach with three traditional methods with real samples under complex background and conduct quantitative comparisons. The effectiveness and accuracy of the developed image models are confirmed by expert assessments, as well as by comparative analysis with the known methods in the field of spatial localizations and production-related effects of flaw detection.

Key words: Computer-aided inspection system, Visual flaws, Capacitive touch screens, Image models, Transformation filtering, Filters, Imaging, Inspection

\section{INTRODUCTION}

According to the growth of smartphones, common touchtone phones are progressively being substituted to arouse a wave of touch screen devices. Touch screens (TSs) are adopted not only for mobile phones but also for computers, televisions, cameras, handheld game consoles and other $3 \mathrm{C}$ products. Thus, the increasing need for TSs encourages the expansion of the TS industry. Currently, touch technology of screen panels can be mainly divided into resistive (an earlier technology), capacitive, optical, electromagnetic, and ultrasonic types. Since resistive touch screens (RTSs) are susceptible to scrape and fire, low light transmittance, and slow response shortcomings, the RTS products cannot satisfy the modern requirements for touch technology and thus have a market share much lower than that of the capacitive touch screens (CTSs). The CTSs, being an unshakable market leader in the TS industry, have the advantages of waterproofness, stain proofness, scratch proofness, fast response, anti-ultraviolet, etc.

The appearance quality of TSs influences their yield rates remarkably in manufacturing process. Lin et al. [1] reported a total flaw rate is $36.2 \%$ of 12 inspection tasks in the manufacturing process of a professional TS producer. Four production operations are related to the visual quality of sensing circuits, inclusive of TS clean, protect film attachment, sensing circuit pre-test and function film attachment. This flaw rate of the four operations is
$14.1 \%$ that is roughly up to $38.95 \%$ of the total flaw rate in the production procedures. Accordingly, automated visual detection for the appearance flaws on the TSs is absolutely essential in the manufacturing process [2, 3].

CTSs are composed of transparent glass substrates, on the surface of which an oxide metal is regularly coated. Figure 1 shows a CTS screen with duplicate modes of sensing circuits and an enlargement of part of a CTS surface. The CTSs have multi-layer structures and are categorized as structural textures. Appearance flaws influence the exteriors of CTSs as well as their durability, reliability and serviceability. It is a severe examination work when flaws are inlaid on appearances of CTSs having structural textures (sensing circuits). Small appearance flaws, regularly arising in the production process of TSs, arouse much more damages and losses when they emerge in electronic components than in manufacturing parts. Thence, to exist in current rival market of sci-tech products, TS producers cannot afford to neglect small appearance flaws $[4,5]$.

The appearance flaws are usually classified into two types: linear and areal. The areal type includes dust, water signs, foams and additional flaws of bigger dimensions. These flaws have the attributes of low contrast, brightness with gradual changes, irregular and non-directional shapes, and occasionally both bright and dark flaws existing at the same time in a region. This kind of flaws compared with the linear type is more complicate to identify its regularity. 


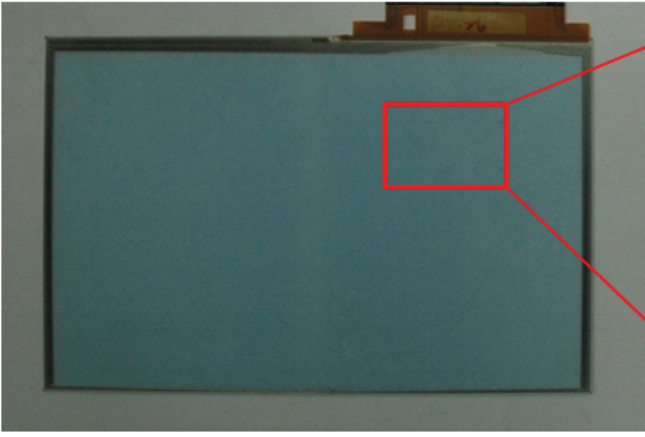

(a)

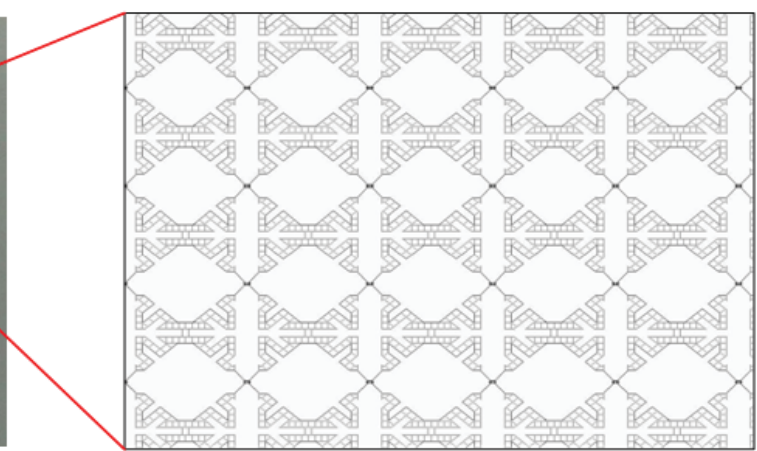

(b)

Figure 1: A CTS display screen (a) with duplicate modes of sensing circuits and (b) an enlargement of part of a CTS surface

Thus, this paper proposes an automated inspection system to detect the areal flaws on TSs. Figure 2 shows a flaw-free image and two defective images with area flaws of CTS appearances having directional repetitive textures. The directional textures indicate grid forms with linear circuits in four ways: horizontal, vertical, and two diagonals. Those background sensing circuits make the flaw detection task harder when area flaws are inlaid in the appearances of directional textures. We thereby propose an image restoration approach based on WT to conquer these problems of automated areal flaw inspection of CTSs.

The rest of the paper is organized as follows: First, we review the literature on optical methods of image processing for flaw detection. Second, we explain the proposed image models for inspecting flaws on CTSs. Third, we conduct the experiments and evaluate the performance of the proposed models with known methods. Four, we present the conclusion and the future work.

\section{LITERATURE REVIEW}

Optical inspection of appearance flaws has turned into an important work for industries who exert to enhance output quality and process efficiency [6, 7]. Flaw inspection techniques compute a set of textural characteristics in a moving mask and seek for crucial partial changes among the feature vectors in spatial or frequency domains [8]. Lin and Chiu [9] developed a machine vision system to find mass centers of chips, locate cutting lines and estimate process regulation plans for the automated and high-speed dicing of electronic passive components. Adamo et al. [10] proposed a low-cost inspection system based on the Canny edge detection for online defects assessment in satin glass. Liu et al. [11] presented the method based on watershed transform methods to segment the possible defective regions and extract features of bottle wall by rules.

Automatic thresholding techniques have also been abroad utilized in the machine vision fields for automatic optical detection of flaws [12]. The Otsu method [13] is the representative approach of threshold techniques for common images regarding consistency and appearance measures. It means chosen threshold values maximizing the between-class variances of intensity histogram. It offers satisfying outcomes for thresholding an image having an intensity histogram of bimodal distribution. $\mathrm{Ng}$ [14] modified the Otsu method by choosing optimum threshold values for unimodal and bimodal distributions, and evaluated the effectiveness of this improved technique on flaw inspections. Navarro et al. [15] developed a sensor system with thresholding technology for inspecting blemishes in ship hull surfaces.

Three popular transforms, Fourier, Gabor and wavelet, are ordinary frequency transformations applied in texture analysis [16]. Nasira and Banumathi[17] utilized the Fourier transform and image processing to detect textile
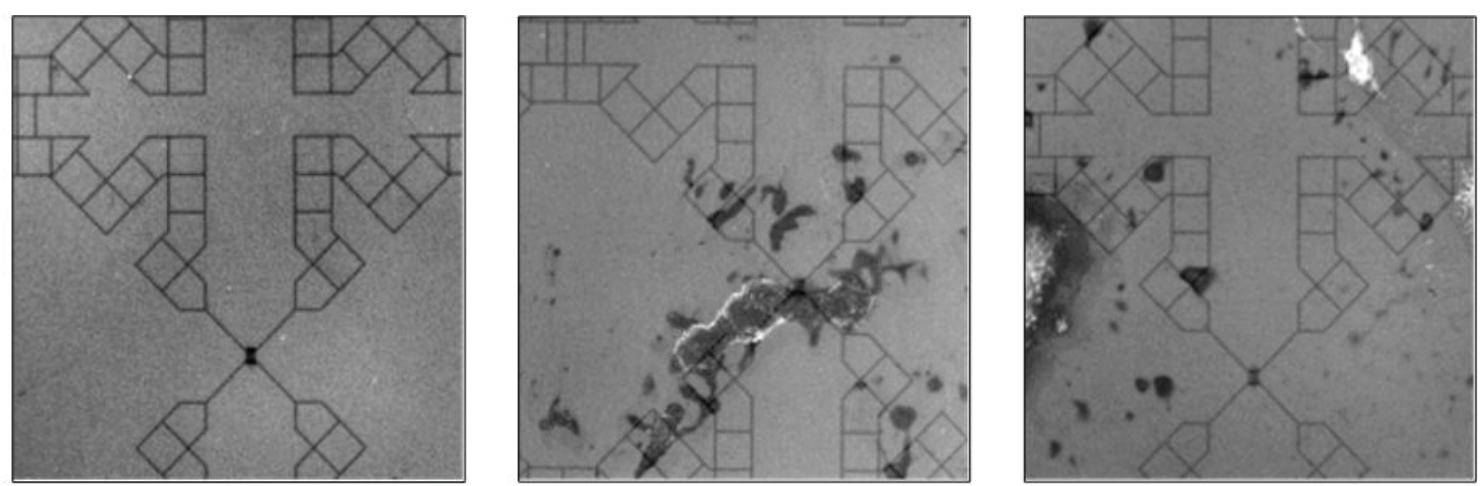

Figure 2: Three CTS images having directional repetitive textures: (a) a flaw-free image; (b) two defective images with various area flaws 
flaws. Tsai and Hsiao [18] developed a wavelet transform method to detect flaws inlaid in uniformly textured surfaces. Lin [19] implemented a wavelet-based multivariate statistical method to detect water flaws with little gray level contrast on the surface of ceramic components. Li [20] used the wavelet transform to strengthen flaw areas and abstract discriminatory characteristics from reconstructed images and the support vector machine to categorize five major flaw classes on copper surfaces. Chang [21] emphasized on structure plan and execution of on-line textile flaw inspection system using Gabor filtering. Lin and Chiu [22] combined block cosine transform and grey relational analysis for blemish detection on domed surfaces in packets of light emitting diodes.

Directional textures with uniform modes are regularly observed on artificial objects, like manufactured parts, cloth textiles, and electronic components. Lu and Tsai [23] presented a machine vision system applying independent component analysis for automatic detection of tiny defects on LCD panel appearances including pure and intricate styles. Regarding flaws detection of TSs, Chen et al. [24] developed an automatic visual inspection device for analogical RTSs. This equipment used image processing techniques to detect flaws on surfaces of RTSs. The RTSs have the pattern of regular spacers in spatial domain and lead to the mode of repeated dots in Fourier domain. Lin and Tsai [25] suggested a Fourier transform based approach to detect linear flaws on CTS appearances. The linear flaws such as scratches and cracks are regular flaws with directional shapes. This kind of flaws compared with the areal type is less complicated to identify its regularity. Hung and Hsieh [2] developed an adaptive model with the ability of learning online to separate defects by using the features of repetitive patterns of the sensing circuits. This approach has a limitation of shadow effect problem that influence on defect detection. Liang et al. [3] proposed a sparse representation-based approach to detect touch screen flaws in low-resolution images. They used a sparsity ratio of the sparse representation coefficients like a measure for distinguishing defective images. The positions of detected defects can only be approximately located. For a new type of compound CTS patterns without primitives nor periodicity, Jiang et al. [5] introduced a combined method of nonnegative matrix factorization with tolerance model for defect detection in such CTS patterns. This method can only be dependably used in such new type of CTS patterns. Therefore, the purpose of this research is to find efficient and effective techniques to automatically detect the appearance flaws on CTSs.

\section{MATERIALS AND METHODS}

This study explores the area flaw detection techniques of the fashionable CTS components. The suggested method transforms a CTS testing image with four distinct ways of regular lines in background to wavelet domain. Then, the directional textures of background will be eliminat- ed by means of filtering the approximate subimage of a breakdown image in wavelet domain. It is hard to exactly inspect area flaws inlaid in intricate directional textures. Consequently, we propose an entire image reconstruction approach applying wavelet transform and flat zone filtering procedure for area flaw inspection on surfaces of CTSs. This technique does not proceed with the procedures of feature extraction and template matching.

\section{Image Modeling\& Analysis}

This study develops a Wavelet Transform (WT) based flat zone filtering approach to detect area flaws of CTSs. The paper extends the earlier work [4] of the authors by a considerable amount of discussion and further experiments. When a CTS image with four distinct ways of line patterns of sensing circuits is transformed to wavelet domain, the directional textures of background can be eliminated by means of filtering the approximate subimage of a break-down wavelet domain image. The filtering scope is determined by a statistical interval. Within the range, the frequency elements will be replaced by the mean value of wavelet frequencies. Subsequently, the filtered image will be reversely transformed to obtain a rebuilt image in spatial domain. Last, the rebuilt image can be easily partitioned into three species (black flaws, gray flaws, and white background) by using a statistical interval estimation method and some characteristics of the identified flaws are abstracted.

\section{Wavelet Transform}

WT offers an easy path to gain a multi-resolution depiction, where texture properties can be likely abstracted. We apply the Haar WT to execute image transformation for frequency filtering since the advantages of Haar WT comprise partial image processing, easy computations, fast processing, memory saving, and numerous image properties [26-28]. The Haar WT is one of the easiest and fundamental WTs. A typical breakdown of a spatial domain image can be performed by early using 1D Haar WT to each row of pixel values and next carrying out another 1D WT to each column. This Haar WT can be calculated step-by-step by the average and half of differences of two adjacent pixels. Due to the transform notion of the 1D WT, the Haar WT could deal with a 2D image of $(\mathrm{M} \times \mathrm{N})$ pixels in the later procedures. The row transfer expresses as:

$$
g_{R}(m, n)=[(g(m, 2 n)+g(m, 2 n+1)) / 2]
$$

$g_{R}(m, n+[Q / 2])=[(g(m, 2 n)-g(m, 2 n+1)) / 2]$

where $0 \leq m \leq(P-1), 0 \leq n \leq[Q / 2]-1$, and [ ] is the Gauss operator. The column transfer denotes as:

$$
\begin{aligned}
& g_{C}(m, n)=\left[\left(g_{R}(2 m, n)+g_{R}(2 m+1, n)\right) / 2\right], \\
& g_{C}(m+[P / 2], n)=\left[\left(g_{R}(2 m, n)-g_{R}(2 m+1, n)\right) / 2\right],
\end{aligned}
$$


where $0 \leq m \leq[P / 2]-1$, and $0 \leq n \leq(Q-1)$.

In the above notations (Eqs. (1-2)), $g(m, n)$ is a testing image, $g_{R}(m, n)$ the row transfer function of $g(m, n)$, and $g_{C}(m, n)$ the column transfer function of $g_{R}(m, n)$. While $g_{c}(m, n)$ is as well the result of the wavelet breakdown of $g(m, n)$, the final results of a Haar WT can be designated as:

$S(m, n)=g_{C}(m, n), T_{1}(m, n)=g_{C}(m, n+[Q / 2])$,

$$
T_{2}(m, n)=g_{C}(m+[P / 2], n), T_{3}(m, n)=g_{C}(m+[P / 2], n+[Q / 2]),
$$

where $0 \leq \mathrm{m} \leq[\mathrm{P} / 2]-1$, and $0 \leq \mathrm{n} \leq[\mathrm{Q} / 2]-1$.

One layer of wavelet breakdown produces one approximate subimage and three detailed subimages including subtle compositions with horizontal, vertical and diagonal directions. An image is broken down by WT into one approximate subimage (S) and three detailed subimages (T1, T2 and T3). The four subimages with sizes of (P/2 $x Q / 2)$ pixels constitute the wavelet features. One-layer Haar wavelet breakdown is utilized to accurately find the pixels with the textural features. Multi-layer wavelet breakdown produces rougher expression of a testing image. A big number of breakdown layers will lead to the mixture effect for the flaws and may arouse localization deviations of the found flaws [29].

\section{Transformation Filtering}

The wavelet approach transforms images into a depiction where presenting spatial and frequency properties. It is appropriate for depicting partial variations in a uniform textured image. For one layer of wavelet breakdown, we gain one approximate subimage and three detailed subimages including subtle compositions with horizontal, vertical and diagonal directions. Through carefully filtering the approximate subimage in distinct breakdown layers for inverse WT, the rebuilt image will eliminate periodical, repeated texture patterns and enhance exclusively regional flaws. A statistical decision interval method can then be applied to distinguish between flaw areas and uniform districts in a rebuilt image. This changes a hard flaw inspection problem in intricate textured images into an easy interval estimation problem in uniform images.

Due to the oscillations of frequency tendency and the properties of low and high frequency districts, we devise a flat zone filter pivoted at the origin of a $2 \mathrm{D}$ wavelet spectrum to filtrate primary low frequency elements of the spectrum image. A suitable band is early selected for the flat zone filter in the WT spectrum. The frequency elements inside the band of the flat zone filter (low frequencies) are then set to the mean value of frequency, and those beyond the filter (medium and high frequencies) are preserved. Last, we transform reversely the filtered WT image back to spatial domain. Selecting an adequate band based on the level of frequency fluctuations for the flat zone filtering task can remarkably intensify the flaw regions in spatial domain. This intensified effects can be obviously viewed in the rebuilt images.

Contrast to ordinary band filtering, the suggested flat zone filtering utilize the mean value of overall frequency instead of zero to substitute the original frequency elements inside the chosen zone area in WT domain. The flat zone filtering process is to decrease the variation between background and texture. The major intention is to eliminate the directional background textures having small changes in intensity, and preserve the area flaws having larger changes in intensity. We use the concept of statistical decision interval to calculate the flat zone area $\bar{x} \pm k \sigma$ and substitute all of the frequency elements inside the region by the frequency mean value $\bar{x}$. The procedure can be expressed as:

$W T(u, v)=\bar{x}$, if $\bar{x}-k \sigma<W T(u, v)<\bar{x}+k \sigma$

$W T(u, v)=W T(u, v)$, otherwise;

where $W T(u, v)$ is a $W T$ frequency image, $\bar{x}$ and $\sigma$ are the mean value and standard deviation of the frequency in $W T(u, v)$, and $\mathrm{k}$ is a constant determined empirically. Figure 3 shows two 3D WT spectrum diagrams which are the before and after plots of the flat zone filtering are executed. Figure $3(a)$ is the $3 D$ diagram of a WT spectrum and Figure $3(\mathrm{~b})$ is the $3 \mathrm{D}$ diagram of a WT spectrum with flat zone filtering process. After the flat zone filtering operation, the frequency elements having larger frequency are intensified in WT domain.

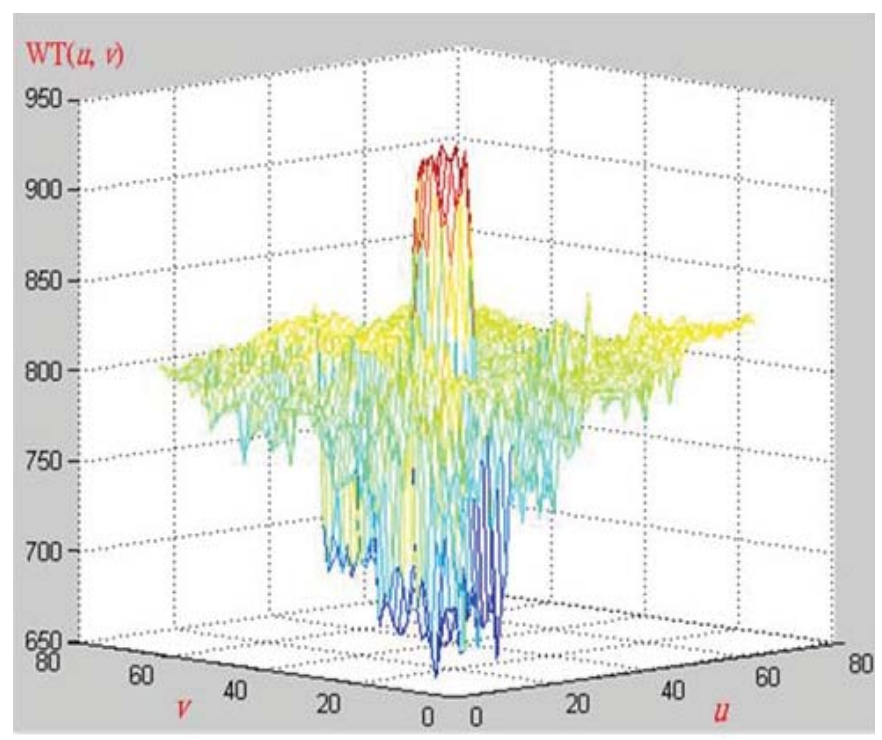

(a)

Figure 3: Two 3-D diagrams of WT spectrum which are before and after plots of the flat zone filtering applied: (a) the $3 D$ diagram of a WT spectrum 


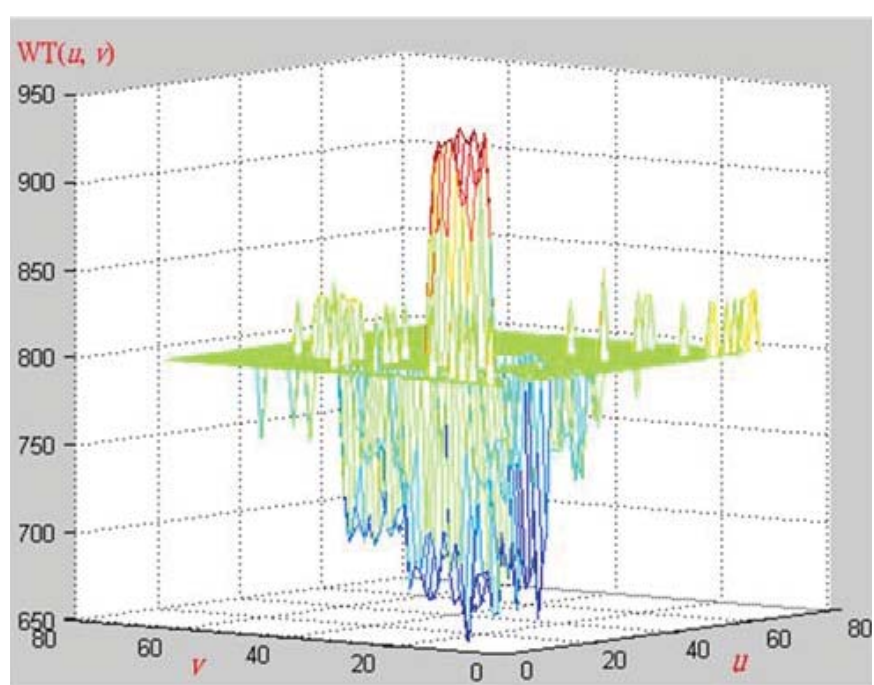

(b)

Figure 3: Two 3-D diagrams of WT spectrum which are before and after plots of the flat zone filtering applied: (b) the 3D diagram of a WT spectrum after flat zone filtering applied

\section{Inverse Transformation and Segmentation}

After the appropriate zone is decided, the frequency filtering procedure can exactly identify the flaw-free low frequency districts and these frequency values are assigned to a mean value in wavelet domain. Then, we conduct reverse WT on the filtered frequency image to spatial domain for flaw segmentation later. In this study, we intend to eliminate most duplicate modes in the rebuilt image by choosing an appropriate zone in the approximate subimages for the mean value replacement. Since structural textures may express high directivity, rebuilding the detailed subimages with directional emphasis distinct from those of the regular textures will delete most directional duplicate modes in an original image, and retain merely partial flaws in a rebuilt image. The directional duplicate modes will lead to a roughly uniform intensity, while the partial flaws will produce different intensities in the rebuilt image.

The rebuilt images have consistent intensities for pixels pertaining to homogeneous background areas, but they also give remarkably distinct intensities for pixels pertaining to heterogeneous flaw regions. The gray level variability in homogeneous areas is small, while the gray level variability in heterogeneous regions is very large compared with the whole rebuilt image. Thence, a statistical method is used to establish an interval for differentiating flaws from duplicate modes in the rebuilt image. The rebuilt image $g^{\prime}(x, y)$ will be roughly a uniform intensity image if a flaw-free testing image is processed. The upper and lower interval limits $\left(L_{L}, L_{u}\right)$ for gray level changes in the rebuilt image are denoted as:

$L=\mu_{g^{\prime}} \pm h \sigma_{g^{\prime}}$ where $L$ is a threshold for separating flaws from background, $\mathrm{h}$ is a control constant, $\mu_{g^{\prime}}$ and $\sigma_{g^{\prime}}$ are a mean and a standard deviation of the rebuilt image with size $M \times M$. The outcome three-level image $G(x, y)$ for flaw segmentation is:

$$
G(x, y)=\left\{\begin{array}{cc}
127 \text { (gray), } & g^{\prime}(x, y)>L_{U} \\
255 \text { (white) }, & L_{L} \leq g^{\prime}(x, y) \leq L_{U} \\
0 \text { (black) }, & g^{\prime}(x, y)<L_{L}
\end{array}\right.
$$

If an intensity value locates in the interval limits $\left(L_{L}, L_{u}\right)$ then gray level is assigned to 255 (white) as a white backdrop. Otherwise, gray level is assigned to 0 (black) as a part of dark flaw if an intensity is less than $L_{L}$ and gray level is assigned to 127 (gray) as a part of gray flaw if an intensity is more than $L_{u}$. Once a pixel with an intensity locates in the interval limits, this pixel is categorized as a homogeneous background component. Otherwise, it is categorized as a heterogeneous flaw component. If the flaw size to be inspected is usually very small compared with a whole testing image, $\mu_{g^{\prime}}$ and $\sigma_{g^{\prime}}$ can be calculated straightly from the rebuilt image of the testing image to adapt lighting changes in the inspection surroundings.

These interval ranges are utilized to differentiate between uniform line modes and flaws in a rebuilt image. The superior and inferior ranges of intensities in a rebuilt image are located at a distance $h \sigma_{g^{\prime}}$ from the mean $\mu_{g^{\prime \prime}}$ Moreover, choosing an appropriate control parameter leads to accurately distinguishing flaws from ordinary areas; however, an inappropriate control parameter outputs numerous incorrectly inspecting ordinary areas as flaws. A little constant value $h$ provides a strict control and can lead to erroneous alerts. A great constant value $\mathrm{h}$ offers a loosen control and can produce lost alerts. A supervised appearance inspection problem is explored in this study. The developed learning systems are ordinary in computer vision and are proper for supervised environments in production sites. The number of WT breakdown layers and the size of flat zone filtering utilized for image rebuild are decided in advance from a texture model. The influence of number of multi-resolution levels and parameter settings of WT filtering process on inspection performance are assessed by trial and error in the experiments.

\section{EXPERIMENTS AND DISCUSSION}

To assess the effect of the suggested method, assessments were carried out on actual CTSs supplied by a TS production plant in Taiwan. All trial samples were arbitrarily chosen from the production process of TSs. Figure 4 illustrates the structures of the exploratory circumstance where we capture a CTS sample to be taken as testing images in the laboratory. The CTS images (270) having thickness $0.78 \mathrm{~mm}$, where 132 have no flaws and 138 have diverse area flaws, were examined in our assessments. All images of the CTS appearance have the same size of $256 \times 256$ pixels and an intensity of 8 bits. 


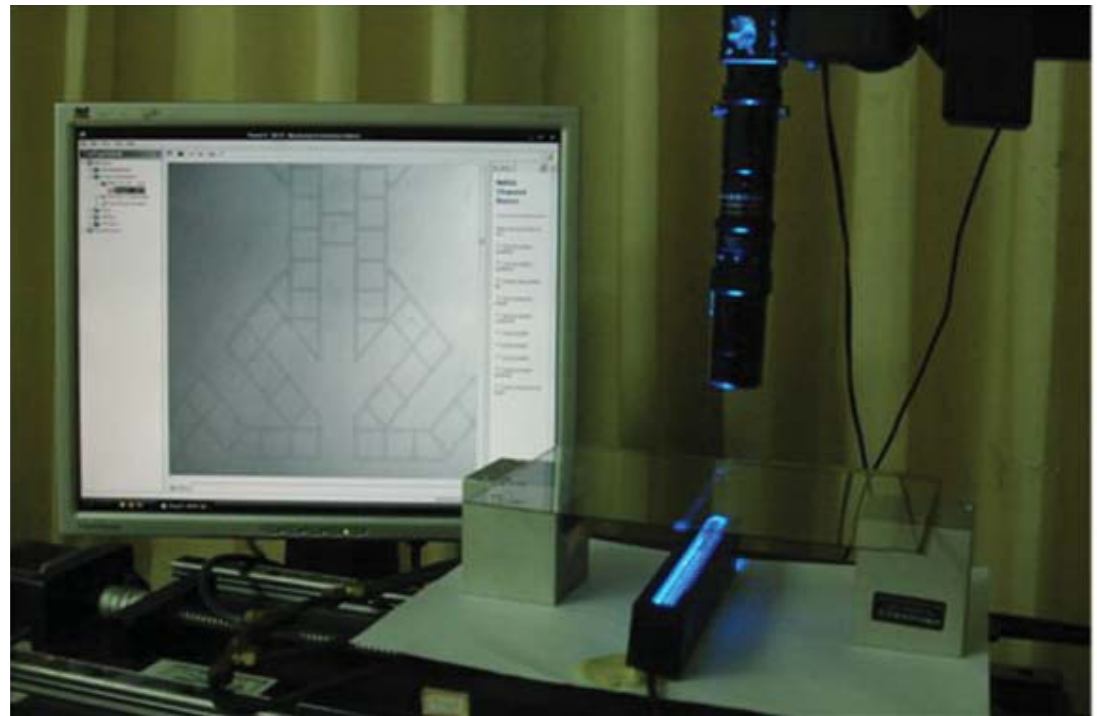

(a)

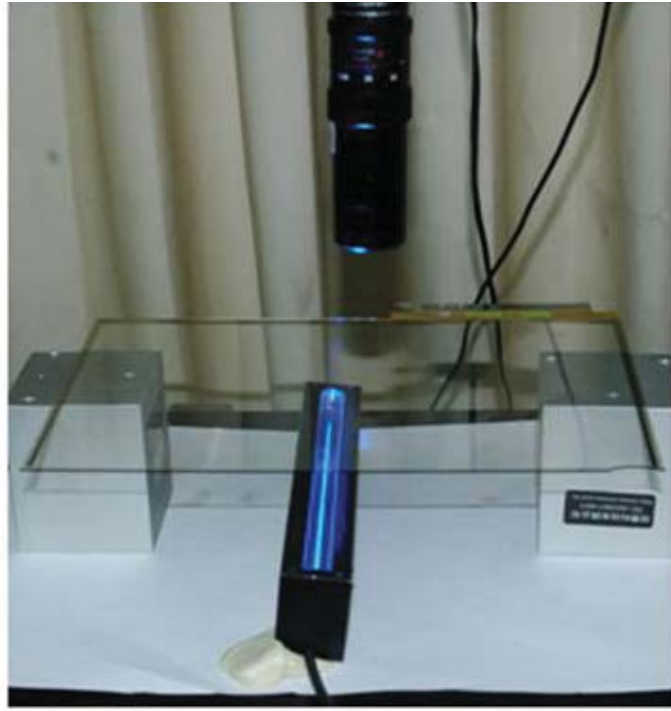

(b)

Figure 4: Structures of the exploratory circumstance where scanning a trial CTS sample: (a) equipment framework of experiments; (b) a trial CTS sample is put on testing platform

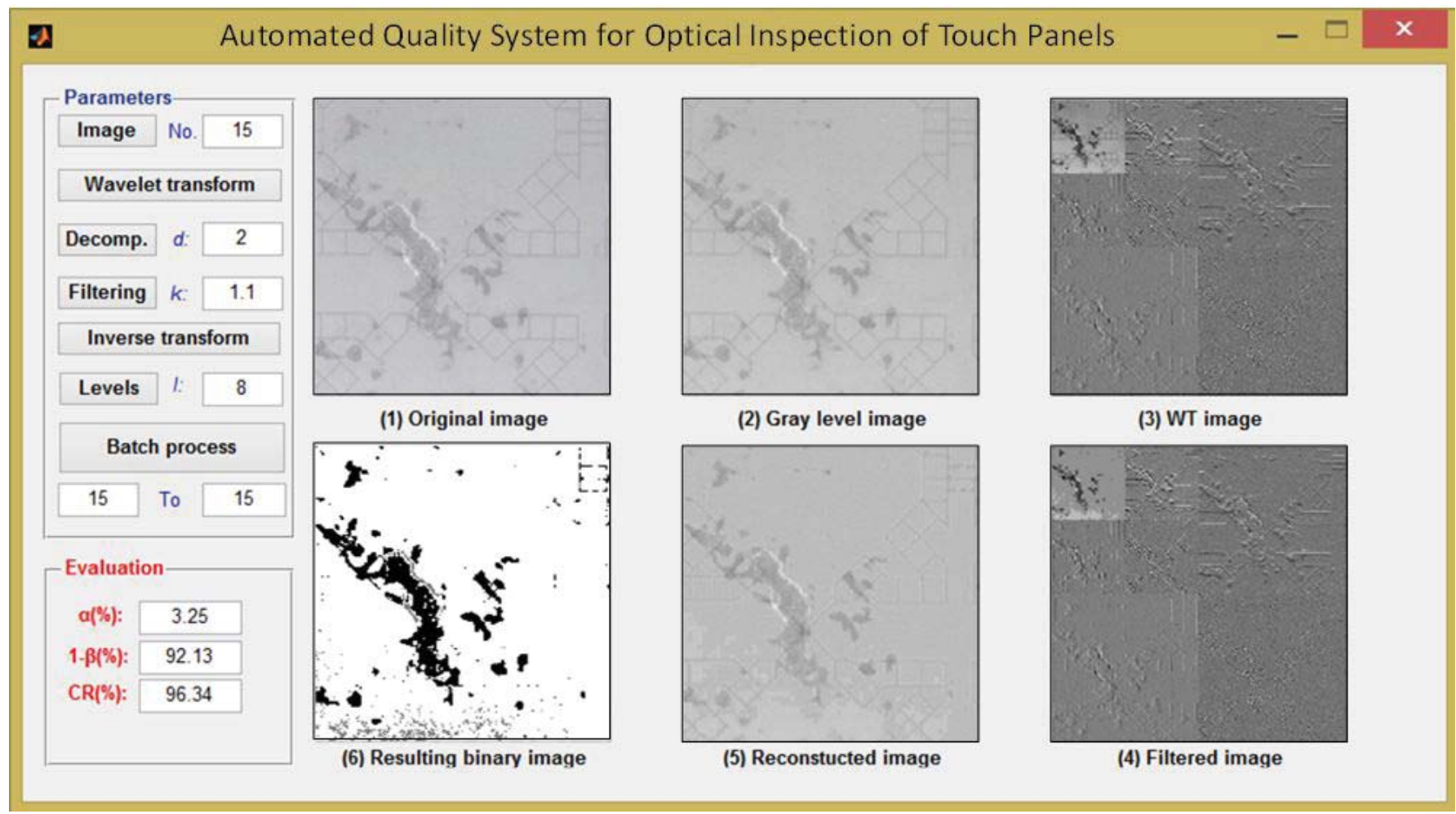

Figure 5: The user interface design of the implemented visual inspection system 
The suggested flaw detection arithmetic is implemented on a personal computer (CPU i5-3230M $2.6 \mathrm{GHz}$ and 4GB RAM) by R2009b version of MATLAB language.

To numerically confirm the manifestation of the suggested method, we contrast the outcomes of our assessments contrary to those supplied by technical assessors (i.e. the ground truth). Three impartial measures: correct classification rate $(C C R)$ and incorrect classification rates ( $\alpha$ and $\beta$ ) were assessed for the effect of the area flaw detection methods. Statistical type I error ( $\alpha)$ measures a probability of generating erroneous alerts, which judge regular areas as flaws. The area of regular regions judged as flaws is divided by the area of real regular regions to gain type I error. Statistical type II error $(\beta)$ measures a probability of generating lost alerts, which invalid to alert actual flaws. The area of undetected flaws is divided by the area of real flaws to gain type II error. The higher the effect assessment measures: (1- $\alpha)$, (1$\beta)$, and $C C R$, the more exact the detection outcomes. The correct classification rate CCR is denoted as:

$\operatorname{CCR}=\left(C_{(1-\alpha)}+C_{(1-\beta)}\right) / C_{\text {total }} * 100 \%$

where $C_{(1-\alpha)}$ is the pixel number of regular regions correctly detected as flaw-free areas, $C_{(1-\beta)}$ is the pixel number of actual flaw regions right detected as flaw areas, and $C_{\text {total }}$ is the pixel number of a testing image.

Figure 5 displays a user interface design of the implemented system. The system interface shows the outcomes and differences executed by the suggested filtering procedure in distinct phases for inspecting area blemishes in CTS appearances. The image (1) and image (2) are a captured image and a corresponding gray scale image from a part of a trial sample. The image (3) is the transformed image of WT with the second breakdown layer and the image (4) depicts the filtered image after the flat zone filtering operation conducted in WT domain. The image (5) and image (6) are the rebuilt image of the filtered image and the resulting three-level images that show the detected black flaws in black and gray flaws in gray by the proposed inspection system, respectively. The outcomes disclose that the area blemishes in CTS appearance are accurately separated in the three-level image, no matter of background with structural patterns.

\section{Choices of important parameters}

WT breakdown of an image with textures in the appropriate level will effectively stress the partial flaws in a consistent appearance. To evaluate the influence of alternating number of breakdown layers on the rebuilt results, experiments present the rebuilt images from breakdown layers 1, 2, 3, and 4, separately. All these images are individually rebuilt from a filtered approximate subimage and three homologous detailed subimages with the Haar wavelet. Both images indicate that very small the number of breakdown layers (e.g. 1 and 2) cannot adequately segment flaws from the repeated texture pat- terns and causes many erroneous alerts. Nevertheless, over large the number of breakdown layers produces the mixed effect of the flaws and results in lots of lost alerts. The number of breakdown layer 3 is more proper to emphasize flaws in the rebuilt image. Our experiments on a diversity of textured images have verified that WT breakdown layer 3 is normally adequate for this area flaw inspection application.

If a decisive thresholding changes, two paired measures, erroneous alert rate $(\alpha)$ and detection rate (1- $\beta$ ), describing the outcomes of a hypothesis testing will vary [30]. When different thresholding are applied, the sets of erroneous alert rates and detection rates are figured as points in a Receiver Operating Characteristic (ROC) curve. The upper-left corner on a ROC figure represents an ideal outcome having $100 \%$ detection rate and a $0 \%$ erroneous alert rate. The more the ROC curve moves toward the upper-left corner,the better the trial executes. High-energy frequency elements related with repetitive line modes may arise approximately the primary zones in wavelet domain images. To remarkably filter out most consistent line modes and entirely retain the area flaws in spatial domain images, the frequency elements on the primary zones must be replaced by the mean value of the frequency elements from the wavelet domain images. The filtering wideness decides the districts of the zone neighborhoods will be filtered out for high-energy frequency elements. Experiments indicate the flaw detection effect of the suggested method with $\mathrm{k}$ value of 1.1 is better than those of the other $k$ values. The flat zone filtering method with larger $k$ value eliminates consistent line modes and partial flaws in the rebuilt image and lead to ignoring small flaws. It suggests the more exact regions of zone neighborhoods are filtered, the better the flaw inspection outcomes will obtain.

\section{Contrast effects of distinct area flaw detection methods}

Three traditional methods commonly applied to defect detection are compared to contrast effects of flaw detection. To reveal the flaw detection outcomes, Figure 6 displays partial outcomes of inspecting area flaws by Otsu method [13], Iterative method [31], three-level method (two threshold values in spatial domain without frequency filtering), the suggested method, and ground truth, separately. The three spatial domain skills, the Otsu, Iterative and three-level methods, make many incorrect alerts on area flaw inspection. The frequency domain skill, the suggested method, inspects majority of area flaws and makes few incorrect alerts. Consequently, the frequency domain technique excels the spatial domain approaches in the area flaw inspection of the CTSs. 


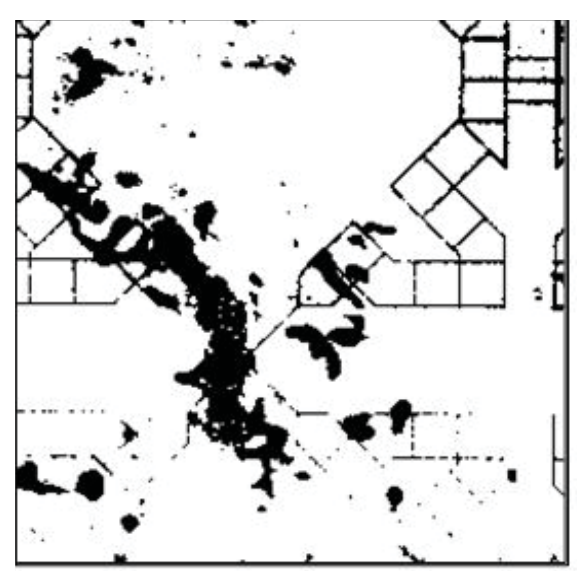

(a)

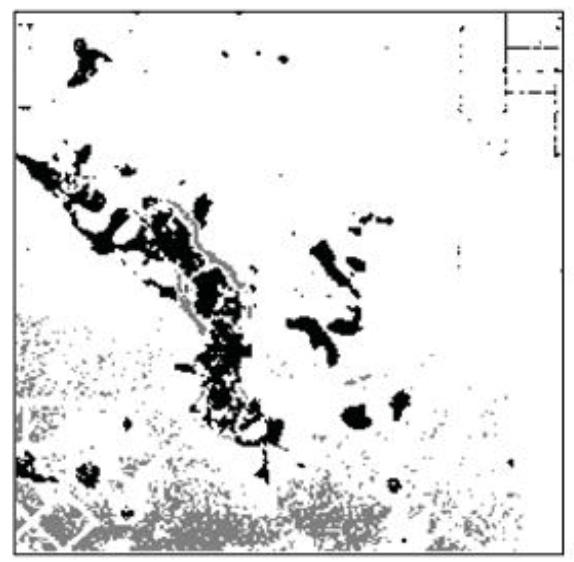

(c)

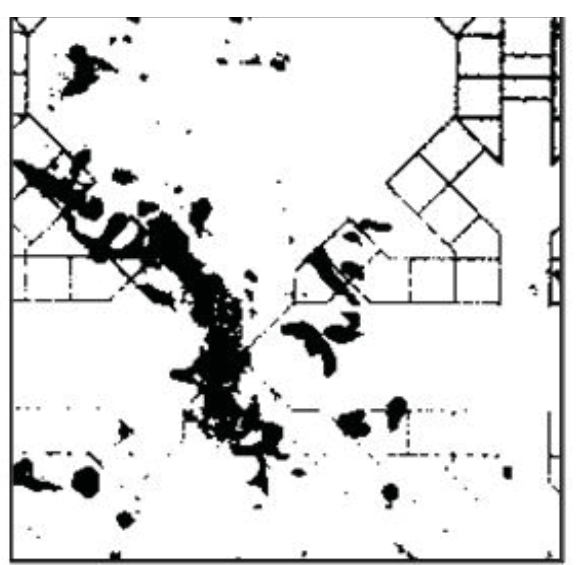

(b)

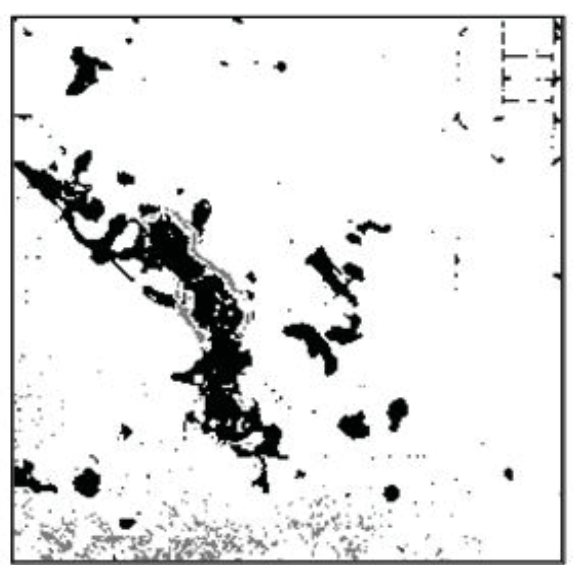

(d)

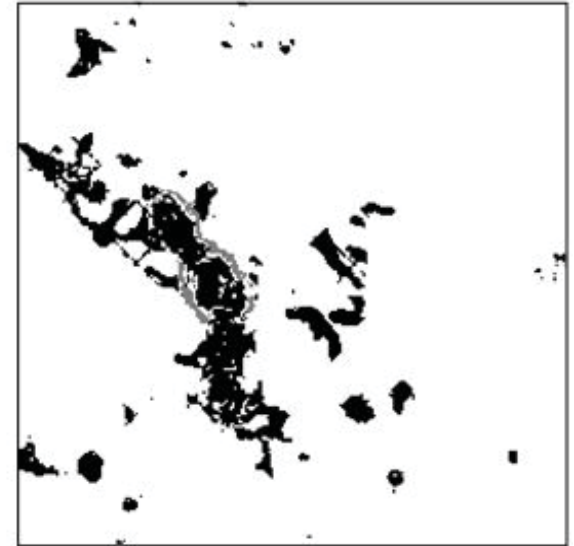

(e)

Figure 6: Resulting examples of area flaw inspection on CTS by: (a) Otsu method, (b) Iterative method,

(c) three-level method, (d) proposed method, and (e) the ground truth

To contrast the effects of different area flaw inspection methods, Table 1 sums up the detection outcomes of our tests. Three spatial domain skills and one frequency domain technique are assessed contrary to the outcomes by technical assessors. The mean flaw detection rates $(1-\beta)$ of all testing images by these methods are, separately, $81.07 \%$ (Otsu method), $80.87 \%$ (Iterative method), $90.14 \%$ (three-level method), and $92.07 \%$ (suggested method). Nevertheless, the three spatial domain skills have remarkably larger erroneous alert rates (a), $26.70 \%$ (Otsu method), $20.41 \%$ (Iterative method), and $11.64 \%$ (three-level method). Otherwise, the suggested approach has quite smaller erroneous alert rate, $4.43 \%$. In addition, the suggested approach has larger
CCR than do the other methods used to area flaw inspection of CTS samples. The average processing time for dealing with a $256 \times 256$ image is: 0.04 seconds by Otsu method, 0.06 seconds by Iterative method, 0.14 seconds by three-level method, and 0.16 seconds by the suggested method. Therefore, the suggested method conquers some troubles of inspecting area flaws on CTSs and outperforms in the ability of accurately differentiating area flaws from textured background.

Table 1: Contrast effects table of four flaw detection methods

\begin{tabular}{|c|c|c|c|c|}
\hline Methods & $\alpha(\%)$ & $1-\beta(\%)$ & CCR (\%) & Time (sec.) \\
\hline Otsu method & 26.70 & 81.07 & 73.46 & 0.04 \\
\hline Iterative method & 20.41 & 80.87 & 79.48 & 0.06 \\
\hline Three - level method & 11.64 & 90.14 & 87.98 & 0.14 \\
\hline Proposed method & 4.43 & 92.07 & 95.32 & 0.16 \\
\hline
\end{tabular}




\section{Experiments on wavelet filtering effects}

To further assess the effect of WT filtering process in the suggested method, experiments with and without the flaw enhancement effect of filtering on CTSs are both executed. All testing images with and without the WT flaw enhancement procedure are separated by the three-level method to investigate how they differ in terms of area flaw inspection. Both of the three-level method and suggested WT-based filtering method achieve good performance in detecting area flaws of CTSs and the appearance flaws that hurt the product quality. Table 2 illustrates the production-related effects of appearance flaw detections by the present method, three-level method, and suggested method. The merits of the suggested method are fourfold: (1).Both of the three-level and suggested methods excel in the ability of accurately discriminating area flaws from ordinary areas. The suggested method has smaller erroneous alert rate and larger flaw detection rate than do the traditional methods. (2). The two methods have larger CCRs than do the present method (human visual inspection) used to area flaw detection of CTS surfaces. (3). The retest rates for detecting appearance flaws of CTSs are as follows: $20.6 \%$ by present method, $7.7 \%$ by three-level method, and $2.6 \%$ by suggested method. The retest rate is the percentage of reinspection and retesting of CTSs that have undertaken rework or other corrections. The suggested method has separately almost three times and eight times smaller average retest rate than do the three-level method and present method. (4).The suggested method has the smallest material wastage rate $3.2 \%$ than do the present inspection method and three-level method because of the excellent flaw detection accuracy. The contrast outcomes of the experiments evidently illustrate the feasible and adequate property of the suggested WTbased filtering method in inspecting area flaws on CTSs.

\section{CONCLUSION}

This study is devoted to the current scientific and technical research. We first reviewthe literature on optical methods of image processing for defect detection on touch screens and confirm the importance and urgency of the development. Then, we introduce a visual inspection system with image models based on transformation filtering for automatic detection of area flaws on CTS surfaces with structural textures. The duplicated line patterns of four directional textures in the testing images can be simply reduced by detecting the band region of an approximate subimage of a breakdown image in wavelet domain, assigning them to a mean value of frequency elements by the flat zone filter, and taking reverse WT to obtain rebuilt image. In the filtered rebuilt image of a CTSsurface, the periodic line regions will have a roughly uniform intensity distribution, while the flaw regions will be obviously preserved. A statistical interval is consequently estimated to establish the interval limits for differentiating among black flaws, gray flaws, and consistent line pattern background. Thus, the intricate area flaws can be precisely identified by the proposed system. The created methods of digital image processing allow to justify localization of flaws on CTS surfaces. Assessment outcomes indicate the suggested method reaches a larger $92.07 \%$ probability of accurately differentiating flaw areas from regular regions and a smaller $4.43 \%$ probability of mistakenly judging regular regions as flaws on repetitive textured appearances of CTSs.

In the computation time of the suggested method, the time of taking forward and reverse WT requires almost 0.1 second averagely without searching any optimization algorithms for parameter determination in advance. This computation time will be remarkably reduced when the parameter optimization has been executed and the WT is carried out in a hardware chip. Thence, the ways of further study will concentrate on investigating existing techniques to look for the most efficient and effective way for the proposed application.

\section{ACKNOWLEDGEMENTS}

Authors deeply thank the Ministry of Science and Technology of Taiwan for sponsor of this study (under grant no. MOST103-2221-E-324 -036).

Table 2: The production-related effects of appearance flaw detections by the three methods

\begin{tabular}{|c|c|c|}
\hline Methods & Retest rate & Material wastage \\
\hline $\begin{array}{c}\text { Present method } \\
\text { (Human visual inspection) }\end{array}$ & $20.6 \%$ & $16.7 \%$ \\
\hline Three-level method & $7.7 \%$ & $4.8 \%$ \\
\hline Suggested method & $2.6 \%$ & $3.2 \%$ \\
\hline
\end{tabular}

\section{REFERENCES}

1. Lin, Y.K., Huang, C.F., Chang, P.C. (2013). System reliability evaluation of a touch panel manufacturing system with defect rate and reworking. Reliability Engineering and System Safety, 118 (10), 51-60.
2. Hung, M.H., Hsieh, C.H. (2015). A novel algorithm for defect inspection of touch panels. Image and $\mathrm{Vi-}$ sion Computing, 41, 11-25.

3. Liang, L.Q., Li, D., Fu, X., Zhang, W.J. (2016). Touch screen defect inspection based on sparse represen- 
tation in low resolution images.Multimedia Tools and Applications, 75(5), 2655-2666.

4. Lin, H.D., Li, J.M. (2014). Automated area defect inspection of touch panels using computer vision. 2014 Proceedings of the International Conference on Image Processing, Computer Vision, and Pattern Recognition (IPCV 2014), p. 16-22.

5. Jiang, C.C., Quan, Y.M., Lin, X.U. (2016). Defect detection of capacitive touch panel using a nonnegative matrix factorization and tolerance model. Applied Optics, 55(9), 2331-2338.

6. Murphy, J.N., Harris, K.D.,Buriak, J.M. (2015). Automated defect and correlation length analysis of block copolymer thin film nanopatterns.PLoS ONE, 10(7): e0133088.

7. Liu, J., Tang, Z., Zhang, J., Chen, Q., Xu, P., Liu, W. (2016). Visual perception-based statistical modeling of complex grain image for product quality monitoring and supervision on assembly production line. PLoS ONE, 11(3): e0146484.

8. Huang, S.H., Pan, Y.C. (2015). Automated visual inspection in the semiconductor industry: A survey. Computers in Industry, 66, 1-10.

9. Lin, H.D.,Chiu, Y.P. (2010). RBF network and EPC method applied to automated process regulations for passive components dicing, International Journal of Innovative Computing Information and Control, 6(11), 5077-5091.

10. Adamo, F., Attivissimo, F., Nisio, A.Di., Savino, M. (2009). A low-cost inspection system for online defects assessment in satin glass. Measurement, 42, 1304-1311.

11. Liu,H., Wang, Y., Duan, F. (2008).Glass bottle inspector based on machine vision. International Journal of Computer Systems Science and Engineering, 3(3), 162-167.

12. Sezgin, M., Sankur, B. (2004). Survey over image thresholding techniques and quantitative performance evaluation. Journal of Electronic Imaging, 13(1), 146-156.

13. Otsu, N. (1979). A threshold selection method from gray level histogram. IEEE Transactions on Systems, Man and Cybernetics, 9, 62-66.

14. Ng, H. F. (2006). Automatic thresholding for defect detection. Pattern Recognition Letters, 27, 16441649.

15. 15) Navarro, P., Iborra, A., Fernández, C., Sánchez, P., Suardíaz, J. (2010). A sensor system for detection of hull surface defects. Sensors, 10, 7067-7081.

16. Gonzalez, R.C., Woods, R.E. (2008). Digital Image Processing. 3rd Ed., Prentice Hall, New Jersey, USA.

17. Nasira, G.M., Banumathi, P. (2013). Fourier transform and image processing in automated fabric defect inspection system. International Journal of Computational Intelligence and Informatics, 3(1), 61-64.

18. Tsai, D.M., Hsiao, B. (2001). Automatic surface inspection using wavelet reconstruction. Pattern Rec- ognition, 34, 1285-1305.

19. Lin, H.D. (2007). Automated visual inspection of ripple defects using wavelet characteristic based multivariate statistical approach. Image and Vision Computing, 25, 1785-1801.

20. Li, T.S. (2009). Applying wavelets transform and support vector machine for copper clad laminate defects classification. Computers \& Industrial Engineering, 56, 1154-1168.

21. Chang, H.F. (2012). Design and implementation of real-time fabric defect detection system. Advances in information Sciences and Service Sciences, 4(21), 23-30.

22. Lin, H.D., Chiu, S.W. (2011). Flaw detection of domed surfaces in LED packages by machine vision system. Expert Systems with Applications, 38, 15208-15216

23. Lu, C.J., Tsai, D.M. (2008). Independent component analysis-based defect detection in patterned liquid crystal display surfaces. Image and Vision Computing, 26, 955-970.

24. Chen, Y.C., Yu, J.H.,Xie, M.C.,Shiou, F.J. (2011). Automated optical inspection system for analogical resistance type touch panel. International Journal of the Physical Sciences, 6(22), 5141-5152.

25. Lin, H.D., Tsai, H.H. (2012). Automated quality inspection of surface defects on touch panels. Journal of the Chinese Institute of Industrial Engineers, 29(5), 291-302.

26. Mallat, S.G. (1989). A theroyfor mulitiresolutionsignal decomposition the wavelet representation. IEEE Transactions on Pattern Analysis and Machine Intelligence, 11(7), 674-693.

27. Shirazi, M.N., Noda, H., Takao, N. (2000). Texture classification based on Markov modeling in wavelet feature space. Image and Vision Computing, 18, 967-973.

28. Arivazhagan, S.,Ganesan, L. (2003). Texture segmentation using wavelet transform. Pattern recognition Letters, 24, 3197-3203.

29. Tsai, D.M., Chiang, C.H. (2003). Automated band selection for wavelet reconstruction in the application of defect detection. Image and Vision Computing, 21, 413-431.

30. Montgomery, D.C. (2009).Statistical Quality Control: A Modern Introduction,6th Edition, John Wiley \& Sons, New York, NY, USA.

31. Nixon, M.S., Aguado, A.S. (2008). Feature Extraction and Image Processing.2nd Edition, Academic Press, Elsevier, Oxford, UK.

Paper submitted: 19.03.2018.

Paper accepted: 04.07.2018.

This is an open access article distributed under the CC BY-NC-ND 4.0 terms and conditions. 